\title{
Analytical relation between the Polyakov loop and Dirac eigenvalues in temporally odd-number lattice QCD
}

\author{
Hideo Suganuma*, Takahiro M. Doi \\ Department of Physics \& Division of Physics and Astronomy, Graduate School of Science, \\ Kyoto University, Kitashirakawaoiwake, Sakyo, Kyoto 606-8502, Japan \\ E-mail: suganuma@scphys.kyoto-u.ac.jp
}

\section{Takumi Iritani}

High Energy Accelerator Research Organization (KEK), Tsukuba, Ibaraki 305-0801, Japan

\begin{abstract}
We derive an analytical gauge-invariant relation between the Polyakov loop $\left\langle L_{P}\right\rangle$ and the Dirac eigenvalues $\lambda_{n}$ in QCD, i.e., $\left\langle L_{P}\right\rangle \propto \sum_{n} \lambda_{n}^{N_{t}-1}\left\langle n\left|\hat{U}_{4}\right| n\right\rangle$, on a temporally odd-number lattice, where the temporal lattice size $N_{t}$ is odd. Here, we use an ordinary square lattice with the normal (nontwisted) periodic boundary condition for link-variables in the temporal direction. This relation is a Dirac spectral representation of the Polyakov loop in terms of Dirac eigenmodes $|n\rangle$. Because of the factor $\lambda_{n}^{N_{t}-1}$ in the Dirac spectral sum, this analytical relation indicates negligibly small contribution of low-lying Dirac modes to the Polyakov loop in both confined and deconfined phases, while the low-lying Dirac modes are essential for chiral symmetry breaking. Also, we numerically confirm the analytical relation, non-zero finiteness of $\left\langle n\left|\hat{U}_{4}\right| n\right\rangle$, and tiny contribution of low-lying Dirac modes to the Polyakov loop in lattice QCD simulations. Thus, we conclude that low-lying Dirac modes are not essential modes for confinement, and there is no direct one-to-one correspondence between confinement and chiral symmetry breaking in QCD.
\end{abstract}

The 31st International Symposium on Lattice Field Theory - LATTICE 2013

July 29 - August 3, 2013

Mainz, Germany

\footnotetext{
* Speaker.
} 


\section{Introduction: color confinement and chiral symmetry breaking}

Quantum chromodynamics (QCD) has two outstanding nonperturbative phenomena of spontaneous chiral-symmetry breaking [1] and color confinement [2 3]. However, their relation is not yet known directly from QCD, and to clarify their precise relation is one of the important problems in theoretical physics 44,5,6,7,8,9.

For chiral symmetry breaking, the standard order parameter is the chiral condensate $\langle\bar{q} q\rangle$, and low-lying Dirac modes play the essential role, as is indicated by the Banks-Casher relation [10]. For quark confinement, the Polyakov loop $\left\langle L_{P}\right\rangle$ is one of the typical order parameters, and it relates to the single-quark free energy $E_{q}$ as $\left\langle L_{P}\right\rangle \propto e^{-E_{q} / T}$ at temperature $T$. The Polyakov loop is the order parameter of spontaneous breaking of the $Z_{N_{c}}$ center symmetry in QCD [11].

A strong correlation between confinement and chiral symmetry breaking has been suggested by the simultaneous phase transitions of deconfinement and chiral restoration in lattice QCD both at finite temperatures and in a finite-volume box [11]. Their strong correlation has been also suggested in terms of QCD-monopoles [4 5], which topologically appear in QCD in the maximally Abelian gauge [2]. Actually, by removing the monopoles from the QCD vacuum, confinement and chiral symmetry breaking are simultaneously lost in lattice QCD [5], which indicates an important role of QCD-monopoles to both confinement and chiral symmetry breaking, and thus these two phenomena seem to be related via the monopole.

In our previous studies 17,9 , aiming to know the direct relation between confinement and chiral symmetry breaking, we study confinement in terms of Dirac eigenmodes in QCD, because low-lying Dirac modes are essential for chiral symmetry breaking [10]. Based on completeness of the Dirac-mode basis, we consider Dirac-mode expansion and projection where the Dirac-mode space is restricted, and investigate the role of low-lying Dirac modes to confinement in SU(3) lattice QCD [7 8, 9]. Remarkably, even after removing the coupling to the low-lying Dirac modes, the following numerically lattice-QCD results are obtained:

1. The Wilson loop obeys the area law [7, , which indicates quark confinement.

2. The string tension, i.e., the confining force, is almost unchanged [7] 8 .

3. The Polyakov loop remains to be almost zero [9], indicating $Z_{3}$-unbroken confinement phase. In fact, quark confinement is kept even in the absence of the low-lying Dirac modes.

In this study, we derive an analytical relation between the Polyakov loop and the Dirac modes in temporally odd-number lattice $\mathrm{QCD}$, where the temporal lattice size is odd, and discuss the relation between confinement and chiral symmetry breaking.

\section{Lattice QCD formalism: general arguments}

To begin with, we state the setup condition of lattice QCD formalism adopted in this study. We use an ordinary square lattice with spacing $a$ and size $N_{s}^{3} \times N_{t}$. The normal (nontwisted) periodic boundary condition is used for the link-variable $U_{\mu}(s)=\mathrm{e}^{i a g A_{\mu}(s)}$ in the temporal direction, with the gluon field $A_{\mu}(s)$, the gauge coupling $g$ and the site $s$. (This temporal periodicity is physically required at finite temperature.) In this paper, we take $\mathrm{SU}\left(N_{c}\right)\left(N_{c}\right.$ : color number) as the gauge group of the theory. However, arbitrary gauge group $G$ can be taken for most arguments in the following. 


\subsection{Dirac operator, Dirac eigenvalues and Dirac modes in lattice QCD}

In lattice QCD, the Dirac operator $\not D=\gamma_{\mu} D_{\mu}$ is expressed with $U_{\mu}(s)=\mathrm{e}^{i a g A_{\mu}(s)}$ as

$$
\not D_{s, s^{\prime}} \equiv \frac{1}{2 a} \sum_{\mu=1}^{4} \gamma_{\mu}\left[U_{\mu}(s) \delta_{s+\hat{\mu}, s^{\prime}}-U_{-\mu}(s) \delta_{s-\hat{\mu}, s^{\prime}}\right]
$$

where $\hat{\mu}$ is the unit vector in $\mu$-direction in the lattice unit, and $U_{-\mu}(s) \equiv U_{\mu}^{\dagger}(s-\hat{\mu})$. Adopting hermitian $\gamma$-matrices as $\gamma_{\mu}^{\dagger}=\gamma_{\mu}$, the Dirac operator $\not D$ is anti-hermitian and satisfies $\not D_{s^{\prime}, s}^{\dagger}=-\not D_{s, s^{\prime}}$. We introduce the normalized Dirac eigen-state $|n\rangle$ as

$$
\not D|n\rangle=i \lambda_{n}|n\rangle, \quad\langle m \mid n\rangle=\delta_{m n},
$$

with the Dirac eigenvalue $i \lambda_{n}\left(\lambda_{n} \in \mathbf{R}\right)$. Because of $\left\{\gamma_{5}, \not D\right\}=0$, the state $\gamma_{5}|n\rangle$ is also an eigen-state of $\not D$ with the eigenvalue $-i \lambda_{n}$. Here, the Dirac eigen-state $|n\rangle$ satisfies the completeness of

$$
\sum_{n}|n\rangle\langle n|=1
$$

For the Dirac eigenfunction $\psi_{n}(s) \equiv\langle s \mid n\rangle$, the explicit form of the Dirac eigenvalue equation $\not D \psi_{n}(s)=i \lambda_{n} \psi_{n}(s)$ in lattice QCD is written by

$$
\frac{1}{2 a} \sum_{\mu=1}^{4} \gamma_{\mu}\left[U_{\mu}(s) \psi_{n}(s+\hat{\mu})-U_{-\mu}(s) \psi_{n}(s-\hat{\mu})\right]=i \lambda_{n} \psi_{n}(s) .
$$

The Dirac eigenfunction $\psi_{n}(s)$ can be numerically obtained in lattice QCD, besides a phase factor. By the gauge transformation of $U_{\mu}(s) \rightarrow V(s) U_{\mu}(s) V^{\dagger}(s+\hat{\mu}), \psi_{n}(s)$ is gauge-transformed as

$$
\psi_{n}(s) \rightarrow V(s) \psi_{n}(s),
$$

which is the same as that of the quark field, although, to be strict, there can appear an irrelevant $n$-dependent global phase factor $e^{i \varphi_{n}[V]}$, according to arbitrariness of the phase in the basis $|n\rangle$ [7].

Note that the spectral density $\rho(\lambda)$ of the Dirac operator $\not D$ relates to chiral symmetry breaking. For example, from Banks-Casher's relation [10], the zero-eigenvalue density $\rho(0)$ leads to $\langle\bar{q} q\rangle$ as

$$
\langle\bar{q} q\rangle=-\lim _{m \rightarrow 0} \lim _{\text {phys }} \rightarrow \infty \rho(0), \quad \rho(\lambda) \equiv \frac{1}{V_{\text {phys }}} \sum_{n}\left\langle\delta\left(\lambda-\lambda_{n}\right)\right\rangle,
$$

with space-time volume $V_{\text {phys }}$. Thus, the low-lying Dirac modes can be regarded as the essential modes responsible to spontaneous chiral-symmetry breaking in QCD.

\subsection{Operator formalism in lattice QCD}

Now, we present the operator formalism in lattice QCD [7, 8, 9. To begin with, we introduce the link-variable operator $\hat{U}_{ \pm \mu}$ defined by the matrix element of

$$
\left\langle s\left|\hat{U}_{ \pm \mu}\right| s^{\prime}\right\rangle=U_{ \pm \mu}(s) \delta_{s \pm \hat{\mu}, s^{\prime}}
$$

With the link-variable operator, the Dirac operator and covariant derivative are simply expressed as

$$
\hat{\not}=\frac{1}{2 a} \sum_{\mu=1}^{4} \gamma_{\mu}\left(\hat{U}_{\mu}-\hat{U}_{-\mu}\right), \quad \hat{D}_{\mu}=\frac{1}{2 a}\left(\hat{U}_{\mu}-\hat{U}_{-\mu}\right) .
$$


The Polyakov loop is also simply written as the functional trace of $\hat{U}_{4}^{N_{t}}$,

$$
\left\langle L_{P}\right\rangle=\frac{1}{N_{c} V}\left\langle\operatorname{Tr}_{c}\left\{\hat{U}_{4}^{N_{t}}\right\}\right\rangle=\frac{1}{N_{c} V}\left\langle\sum_{s} \operatorname{tr}_{c}\left(\prod_{n=0}^{N_{t}-1} U_{4}(s+n \hat{t})\right)\right\rangle,
$$

with the four-dimensional lattice volume $V \equiv N_{s}^{3} \times N_{t}$ and $\hat{t}=\hat{4}$. Here, " $\operatorname{Tr}_{c}$ " denotes the functional trace of $\operatorname{Tr}_{c} \equiv \sum_{s} \operatorname{tr}_{c}$ with the trace $\operatorname{tr}_{c}$ over color index.

The Dirac-mode matrix element of the link-variable operator $\hat{U}_{\mu}$ can be expressed with $\psi_{n}(s)$ :

$$
\left\langle m\left|\hat{U}_{\mu}\right| n\right\rangle=\sum_{s}\langle m \mid s\rangle\left\langle s\left|\hat{U}_{\mu}\right| s+\hat{\mu}\right\rangle\langle s+\hat{\mu} \mid n\rangle=\sum_{s} \psi_{m}^{\dagger}(s) U_{\mu}(s) \psi_{n}(s+\hat{\mu}) .
$$

Note that the matrix element is gauge invariant, apart from an irrelevant phase factor. Actually, using the gauge transformation [2.5], we find the gauge transformation of the matrix element as [7]

$$
\begin{aligned}
\left\langle m\left|\hat{U}_{\mu}\right| n\right\rangle & =\sum_{s} \psi_{m}^{\dagger}(s) U_{\mu}(s) \psi_{n}(s+\hat{\mu}) \rightarrow \sum_{s} \psi_{m}^{\dagger}(s) V^{\dagger}(s) \cdot V(s) U_{\mu}(s) V^{\dagger}(s+\hat{\mu}) \cdot V(s+\hat{\mu}) \psi_{n}(s+\hat{\mu}) \\
& =\sum_{s} \psi_{m}^{\dagger}(s) U_{\mu}(s) \psi_{n}(s+\hat{\mu})=\left\langle m\left|\hat{U}_{\mu}\right| n\right\rangle .
\end{aligned}
$$

To be strict, there appears an $n$-dependent global phase factor, corresponding to the arbitrariness of the phase in the basis $|n\rangle$. However, this phase factor cancels as $e^{i \varphi_{n}} e^{-i \varphi_{n}}=1$ between $|n\rangle$ and $\langle n|$, and does not appear for physical quantities such as the Wilson loop and the Polyakov loop [7].

\section{Analytical relation between the Polyakov loop and Dirac modes in temporally odd-number lattice QCD}

Now, we consider a temporally odd-number lattice, where the temporal lattice size $N_{t}$ is odd. Here, we use an ordinary square lattice with the normal (nontwisted) periodic boundary condition for the link-variable in the temporal direction. The spatial lattice size $N_{s}$ is taken to be larger than $N_{t}$, i.e., $N_{s}>N_{t}$. Note that, in the continuum limit of $a \rightarrow 0$ and $N_{t} \rightarrow \infty$, any number of large $N_{t}$ gives the same physical result. Then, in principle, it is no problem to use the odd-number lattice.

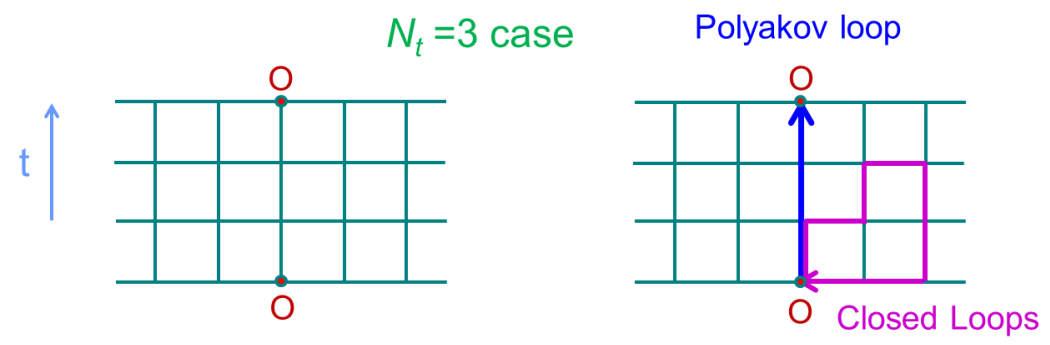

Figure 1: An example of the temporally odd-number lattice. Only gauge-invariant quantities such as closed loops and the Polyakov loop survive in QCD. Closed loops have even-number links on the square lattice.

In general, only gauge-invariant quantities such as closed loops and the Polyakov loop survive in QCD, according to the Elitzur theorem [11]. All the non-closed lines are gauge-variant and their expectation values are zero. Note here that any closed loop (except for the Polyakov loop) needs even-number link-variables on the square lattice. (See Fig.1.) 
On the temporally odd-number lattice, we consider the functional trace of

$$
I \equiv \operatorname{Tr}_{c, \gamma}\left(\hat{U}_{4} \hat{\not}^{N_{t}-1}\right),
$$

where $\operatorname{Tr}_{c, \gamma} \equiv \sum_{s} \operatorname{tr}_{c} \operatorname{tr}_{\gamma}$ includes $\operatorname{tr}_{c}$ and the trace $\operatorname{tr}_{\gamma}$ over spinor index. Its expectation value

$$
\langle I\rangle=\left\langle\operatorname{Tr}_{c, \gamma}\left(\hat{U}_{4} \hat{D}^{N_{t}-1}\right)\right\rangle
$$

is obtained as the gauge-configuration average in lattice QCD. In the case of enough large volume $V$, one can expect $\langle O\rangle \simeq \operatorname{Tr} O / \operatorname{Tr} 1$ for any operator $O$ at each gauge configuration.

From Eq. (2.8), $\hat{U}_{4} \hat{D}^{N_{t}-1}$ is expressed as a sum of products of $N_{t}$ link-variable operators, since the Dirac operator $\hat{\not D}$ includes one link-variable operator in each direction of $\pm \mu$. Then, $\hat{U}_{4} \hat{D}^{N_{t}-1}$ includes many trajectories with the total length $N_{t}$ in the lattice unit on the square lattice, as shown in Fig.2. Note that all the trajectories with the odd-number length $N_{t}$ cannot form a closed loop on the square lattice, and thus give gauge-variant contribution, except for the Polyakov loop.

$N_{t}=3$ case

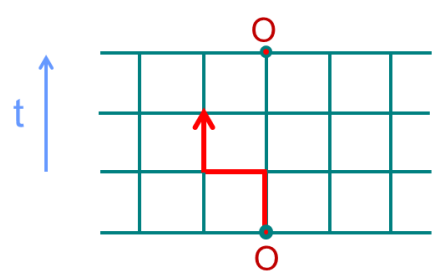

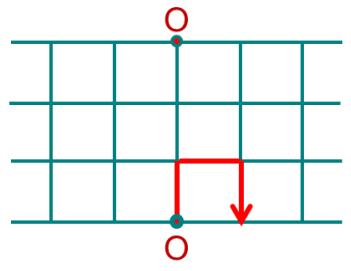

Gauge-variant

Figure 2: A part of the trajectories stemming from $\left\langle\operatorname{Tr}_{c, \gamma}\left(\hat{U}_{4} \hat{D}^{N_{t}-1}\right)\right\rangle$. For each trajectory, the first step is positive temporal direction, and the total length is $N_{t}$. All the trajectories with the odd-number length $N_{t}$ cannot form a closed loop on the square lattice, and thus gauge-variant, except for the Polyakov loop.

Hence, among the trajectories stemming from $\left\langle\operatorname{Tr}_{c, \gamma}\left(\hat{U}_{4} \hat{\not}^{N_{t}-1}\right)\right\rangle$, all the non-loop trajectories are gauge-variant and give no contribution, according to the Elitzur theorem. Only the exception is the Polyakov loop. (See Figs.2 and 3.) [For each trajectory in $\hat{U}_{4} \hat{D}^{N_{t}-1}$, the first step is positive temporal direction corresponding to $\hat{U}_{4}$, so that $\left\langle\operatorname{Tr}_{c, \gamma}\left(\hat{U}_{4} \hat{D}^{N_{t}-1}\right)\right\rangle$ cannot include the anti-Polyakov loop $\left\langle L_{P}^{\dagger}\right\rangle$.] Thus, in the functional trace $\langle I\rangle=\left\langle\operatorname{Tr}_{c, \gamma}\left(\hat{U}_{4} \hat{D}^{N_{t}-1}\right)\right\rangle$, only the Polyakov-loop ingredient can survive as the gauge-invariant quantity, and $\langle I\rangle$ is proportional to the Polyakov loop $\left\langle L_{P}\right\rangle$.

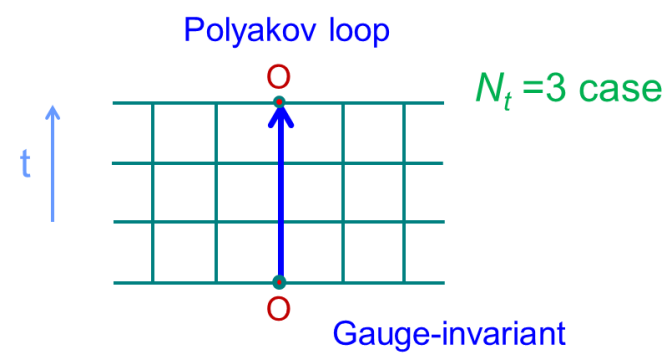

Figure 3: Among the trajectories stemming from $\left\langle\operatorname{Tr}_{c, \gamma}\left(\hat{U}_{4} \hat{D}^{N_{t}-1}\right)\right\rangle$, only the Polyakov-loop ingredient can survive as the gauge-invariant quantity. Owing to the first factor $\hat{U}_{4},\left\langle\operatorname{Tr}_{c, \gamma}\left(\hat{U}_{4} \hat{D}^{N_{t}-1}\right)\right\rangle$ does not include $\left\langle L_{P}^{\dagger}\right\rangle$. 
Actually, we can mathematically derive the following relation:

$$
\begin{aligned}
\langle I\rangle & =\left\langle\operatorname{Tr}_{c, \gamma}\left(\hat{U}_{4} \hat{D}^{N_{t}-1}\right)\right\rangle & & \\
& =\left\langle\operatorname{Tr}_{c, \gamma}\left\{\hat{U}_{4}\left(\gamma_{4} \hat{D}_{4}\right)^{N_{t}-1}\right\}\right\rangle & & (\because \text { only gauge-invariant terms survive }) \\
& =4\left\langle\operatorname{Tr}_{c}\left(\hat{U}_{4} \hat{D}_{4}^{N_{t}-1}\right)\right\rangle & & \left(\because \gamma_{4}^{N_{t}-1}=1, \operatorname{tr}_{\gamma} 1=4\right) \\
& =\frac{4}{(2 a)^{N_{t}-1}}\left\langle\operatorname{Tr}_{c}\left\{\hat{U}_{4}\left(\hat{U}_{4}-\hat{U}_{-4}\right)^{N_{t}-1}\right\}\right\rangle & & \left(\because \hat{D}_{4}=\frac{1}{2 a}\left(\hat{U}_{4}-\hat{U}_{-4}\right)\right) \\
& =\frac{4}{(2 a)^{N_{t}-1}}\left\langle\operatorname{Tr}_{c}\left\{\hat{U}_{4}^{N_{t}}\right\}\right\rangle & & (\because \text { only gauge-invariant terms survive }) \\
& =\frac{12 V}{(2 a)^{N_{t}-1}}\left\langle L_{P}\right\rangle . & &
\end{aligned}
$$

We thus obtain the relation between $\langle I\rangle=\left\langle\operatorname{Tr}_{c, \gamma}\left(\hat{U}_{4} \hat{\not}^{N_{t}-1}\right)\right\rangle$ and the Polyakov loop $\left\langle L_{P}\right\rangle$,

$$
\langle I\rangle=\left\langle\operatorname{Tr}_{c, \gamma}\left(\hat{U}_{4} \hat{\not}^{N_{t}-1}\right)\right\rangle=\frac{12 V}{(2 a)^{N_{t}-1}}\left\langle L_{P}\right\rangle .
$$

On the other hand, we calculate the functional trace in Eq.(3.2) using the complete set of the Dirac-mode basis $|n\rangle$ satisfying $\sum_{n}|n\rangle\langle n|=1$, and find the Dirac-mode representation of

$$
\langle I\rangle=\sum_{n}\left\langle n\left|\hat{U}_{4} \hat{D}^{N_{t}-1}\right| n\right\rangle=i^{N_{t}-1} \sum_{n} \lambda_{n}^{N_{t}-1}\left\langle n\left|\hat{U}_{4}\right| n\right\rangle .
$$

Combing Eqs. (3.4) and (3.5), we obtain the analytical relation between the Polyakov loop $\left\langle L_{P}\right\rangle$ and the Dirac eigenvalues $i \lambda_{n}$ :

$$
\left\langle L_{P}\right\rangle=\frac{(2 a i)^{N_{t}-1}}{12 V} \sum_{n} \lambda_{n}^{N_{t}-1}\left\langle n\left|\hat{U}_{4}\right| n\right\rangle
$$

This is a direct relation between the Polyakov loop $\left\langle L_{P}\right\rangle$ and the Dirac modes in QCD, and is mathematically valid on the temporally odd-number lattice in both confined and deconfined phases. The relation (3.6) is a Dirac spectral representation of the Polyakov loop, and we can investigate each Dirac-mode contribution to the Polyakov loop individually, based on Eq. 3.6. (For example, each contribution specified by $n$ is numerically calculable in lattice QCD.) In particular, from Eq.(3.6), we can discuss the relation between confinement and chiral symmetry breaking in QCD.

As a remarkable fact, because of the factor $\lambda_{n}^{N_{t}-1}$, the contribution from low-lying Diracmodes with $\left|\lambda_{n}\right| \simeq 0$ is negligibly small in the Dirac spectral sum of RHS in Eq. 3.6, compared to the other Dirac-mode contribution. In fact, the low-lying Dirac modes have tiny contribution to the Polyakov loop, regardless of confined or deconfined phase.

Here, we give several comments on the relation (3.6) in order.

1) Equation (3.6) is a manifestly gauge-invariant relation. Actually, the matrix element $\left\langle n\left|\hat{U}_{4}\right| n\right\rangle$ can be expressed with the Dirac eigenfunction $\psi_{n}(s)$ and the temporal link-variable $U_{4}(s)$ as

$$
\left\langle n\left|\hat{U}_{4}\right| n\right\rangle=\sum_{s}\langle n \mid s\rangle\left\langle s\left|\hat{U}_{4}\right| s+\hat{t}\right\rangle\langle s+\hat{t} \mid n\rangle=\sum_{s} \psi_{n}^{\dagger}(s) U_{4}(s) \psi_{n}(s+\hat{t})
$$

and each term $\psi_{n}^{\dagger}(s) U_{4}(s) \psi_{n}(s+\hat{t})$ is manifestly gauge invariant, because of the gauge transformation property (2.5). [Global phase factors also cancel exactly as $e^{-i \varphi_{n}} e^{i \varphi_{n}}=1$ between $\langle n|$ and $|n\rangle$.] 
2) In RHS of Eq. (3.6), there is no cancellation between chiral-pair Dirac eigen-states, $|n\rangle$ and $\gamma_{5}|n\rangle$, because $\left(N_{t}-1\right)$ is even, i.e., $\left(-\lambda_{n}\right)^{N_{t}-1}=\lambda_{n}^{N_{t}-1}$, and $\left\langle n\left|\gamma_{5} \hat{U}_{4} \gamma_{5}\right| n\right\rangle=\left\langle n\left|\hat{U}_{4}\right| n\right\rangle$.

3) Even in the presence of a possible multiplicative renormalization factor for the Polyakov loop, the contribution from the low-lying Dirac modes (or the small $\left|\lambda_{n}\right|$ region) is relatively negligible, compared to other Dirac-mode contribution in the sum of RHS in Eq.(3.6).

4) If RHS in Eq. (3.6) were not a sum but a product, low-lying Dirac modes (or the small $\left|\lambda_{n}\right|$ region) should have given an important contribution to the Polyakov loop as a crucial reduction factor of $\lambda_{n}^{N_{t}-1}$. In the sum, however, the contribution $\left(\propto \lambda_{n}^{N_{t}-1}\right)$ from the small $\left|\lambda_{n}\right|$ region is negligible.

5) Even if $\left\langle n\left|\hat{U}_{4}\right| n\right\rangle$ behaves as the $\delta$-function $\delta(\lambda)$, the factor $\lambda_{n}^{N_{t}-1}$ is still crucial in RHS of Eq.(3.6), because of $\lambda \delta(\lambda)=0$.

6) The relation (3.6) is correct regardless of presence or absence of dynamical quarks, although the dynamical quark effect appears in $\left\langle L_{P}\right\rangle$, the Dirac eigenvalue distribution $\rho(\lambda)$ and $\left\langle n\left|\hat{U}_{4}\right| n\right\rangle$.

Note that all the above arguments can be numerically confirmed by lattice QCD calculations. Using actual lattice QCD calculations at the quenched level, we numerically confirm the analytical relation (3.6), non-zero finiteness of $\left\langle n\left|\hat{U}_{4}\right| n\right\rangle$, and the negligibly small contribution of low-lying Dirac modes to the Polyakov loop, in both confined and deconfined phases.

From the analytical relation (3.6) and the numerical confirmation, we conclude that low-lying Dirac-modes have tiny contribution to the Polyakov loop, and are not essential for confinement, while these modes are essential for chiral symmetry breaking. This conclusion indicates no direct one-to-one correspondence between confinement and chiral symmetry breaking in QCD.

\section{Acknowledgements}

H.S. and T.I. are supported in part by the Grant for Scientific Research [(C) No.23540306, E01:21105006, No.21674002] from the Ministry of Education, Science and Technology of Japan.

\section{References}

[1] Y. Nambu and G. Jona-Lasinio, Phys. Rev. 122 (1961) 345; ibid. 124 (1961) 246.

[2] Y. Nambu, Phys. Rev. D10 (1974) 4262; G. 't Hooft, Nucl. Phys. B190 (1981) 455.

[3] A. Yamamoto and H. Suganuma, Phys. Rev. Lett. 101 (2008) 241601; Phys. Rev. D79 (2009) 054504.

[4] H. Suganuma, S. Sasaki and H. Toki, Nucl. Phys. $B 435$ (1995) 207; Prog. Theor. Phys. 94 (1995) 373.

[5] O. Miyamura, Phys. Lett. B353 (1995) 91; R.M. Woloshyn, Phys. Rev. D51 (1995) 6411.

[6] C. Gattringer, Phys. Rev. Lett. 97 (2006) 032003; F. Bruckmann et al., Phys. Lett. $B 647$ (2007) 56.

[7] S. Gongyo, T. Iritani and H. Suganuma, Phys. Rev. D86 (2012) 034510.

[8] H. Suganuma, S. Gongyo, T. Iritani and A. Yamamoto, PoS (QCD-TNT-II) (2011) 044. H. Suganuma, S.Gongyo, T.Iritani, $P o S$ (Lattice 2012) (2012) 217; PoS (Confinement X) (2013) 081.

[9] T. Iritani and H. Suganuma, arXiv:1305.4049[hep-lat]; T. Iritani, S. Gongyo and H. Suganuma, PoS (Lattice 2012) (2012) 218; PoS (Confinement X) (2013) 053.

[10] T. Banks and A. Casher, Nucl. Phys. B169 (1980) 103.

[11] H.-J. Rothe, Lattice Gauge Theories, 4th edition, World Scientific, 2012. 